\title{
Heterogeneous and opportunistic wireless networks
}

Jordi Pérez-Romero ( $\bowtie)$, Sergio Palazzo, Laura Galluccio, Giacomo Morabito, Alessandro Leonardi, Carles Antón-Haro, Erdal Arıkan, Gerd Asheid, Marc Belleville, Ioannis Dagres, Davide Dardari, Mischa Dohler, Yonina Eldar, Laura Galluccio, Sinan Gezici, Lorenza Giupponi, Christine Guillemot, George Iosifidis, Michel Kieffer, Marios Kountouris, Alessandro Leonardi, Marco Luise, Guido Masera, Javier Matamoros, Natalia Miliou, Giacomo Morabito, Dominique Morche, Christophe Moy, Carles Navarro, Gabriella Olmo, Sergio Palazzo, Jacques Palicot, Raffaella Pedone, Venkatesh Ramakrishnan, Shlomo Shamai, Piotr Tyczka, Luc Vandendorpe, Alessandro Vanelli-Coralli, Claudio Weidmann, and Andreas Zalonis

Recent years have witnessed the evolution of a large plethora of wireless technologies with different characteristics, as a response of the operators' and users' needs in terms of an efficient and ubiquitous delivery of advanced multimedia services. The wireless segment of network infrastructure has penetrated in our lives, and wireless connectivity has now reached a state where it is considered to be an indispensable service as electricity or water supply. Wireless data networks grow increasingly complex as a multiplicity of wireless information terminals with sophisticated capabilities get embedded in the infrastructure.

When looking at the horizon of the next decades, even more significant changes are expected, bringing the wireless world closer and closer to our daily life, which will definitely pose new challenges in the design of future wireless networks. In the following sections, a vision is briefly described on some of the envisaged elements that will guide this evolution, and that will definitely impact of the design of the network layers of wireless networks.

In particular, and addressing a shorter term perspective, the paper starts in Section 1 with the implications of network heterogeneity on how wireless networks will be operated and perceived by the users, with the corresponding requirements for smart radio resource management strategies. Then, Section 2 will address the concept of self-organising networks, since it is expected that, due to the increase in complexity in future networks, together with the huge amount of parameters to be tuned for network optimisation, mechanisms are established to allow the self-configuration of a wireless network with minimum human intervention. Going a step further from the more classical cellular networks and their evolutions, the next sections address other types of wireless communications networks that are also envisaged to arise

Benedetto S., Correia L.M., Luise M. (eds.): The Newcom++ Vision Book. Perspectives of Research on Wireless Communications in Europe.

DOI 10.1007/978-88-470-1983-6_3, @ Springer-Verlag Italia 2012 
in the next years. Specifically, Section 3 will be devoted to opportunistic networks, while Section 4 will present the application of wireless technologies in vehicular communication networks and finally Section 5 will be devoted to the Future Internet and the Internet of Things concept, addressing in particular the implications from the wireless perspective. The paper will end in Section 6 with a look towards the long term future by analysing the possibility to extend networking concepts to the nanoscale communications, in the so-called nanonetwork concept, a futuristic interdisciplinary field involving both networking and biological aspects, with still many open research problems but that can generate a new paradigm in how networks can be understood in a time horizon of some decades.

\section{Heterogeneous networks and adaptive RRM}

A clear trend during the last decade has been a very significant increase in the user demand for wireless communications services, moving from classical voice service towards high bit rate demanding data services, including Internet accessibility everywhere and anytime. While it could have appeared that the penetration of wireless data services experienced a slow launching time, like in the case of Internet access through 2.5G networks such as GPRS, maybe one of the main reasons for that was that the achievable bit rates of only some tenths of kb/s were still very low to support a data service with similar characteristics to that of the wired Internet connections. In fact, this trend was completely changed with the appearance of $3 \mathrm{G}$ and particularly 3.5G networks like HSDPA. Thanks to the significantly larger bit rates available, in the order of a few $\mathrm{Mb} / \mathrm{s}$, wireless Internet access was much more attractive for final users, with the corresponding explosion in the usage of devices enabling wireless Internet access (e.g. USB modems, modems integrated in laptops, etc.). In that respect, and given that current applications are more and more bit rate demanding, such as multimedia traffic, games, 3D applications, etc., there is no particular reason to envisage that the trend towards demanding higher bit rates in the wireless segment will change in the short future. In fact, this is the trend that can already be appreciated under the development of future LTE and LTE-Advanced systems, which, as a difference from previous generations like GSM or UMTS, now place the focus on the packet data services while the provision of classical voice services is seen as something rather complementary that will be mainly provided through $2 \mathrm{G} / 3 \mathrm{G}$ legacy networks.

From a general perspective, the provision of wireless communication services requires from the operator perspective to carry out an appropriate network dimensioning and deployment based on the technologies available and in accordance with the expected traffic demand over a certain geographical area. The target should be then to ensure that services are provided under specific constraints in terms of quality observed by the user, related both to accessibility (e.g. coverage area, reduced blocking/dropping probabilities) and to the specific service requirements (e.g. bit rate, delay, etc.), while at the same time ensuring that radio resources are used efficiently so that operator can maximize capacity. Then, when looking at the basic 
ingredients to deal with an increasing demand of traffic and bit rate in wireless communication services, this can mainly come through the following general principles:

- Increasing the spectral efficiency, i.e. the number of bits/s that can be delivered per unit of spectrum. This can be achieved from the technological perspective, improving the transmission mechanisms at the different layers (e.g. efficient access techniques, efficient radio resource management mechanisms, etc.). This trend has also been observed in the past evolution of mobile technologies with the adoption of more efficient multiple access techniques in each mobile generation, (e.g. from TDMA in GSM to CDMA in UMTS and OFDMA in LTE), with the inclusion of adaptive Radio Resource Management (RRM) mechanisms that try to better exploit the different channel conditions perceived by users (e.g. link adaptation strategies, fast packet scheduling, etc.), and with the adoption of MIMO technologies.

- Increasing the number of base stations to provide a service in a given area, thus reducing the coverage area of each one. On the one hand, this allows that all resources available in the base station are used in a smaller area, resulting in a larger efficiency in terms of resources per unit of area. On the other hand, this also allows mobile users being connected closer to their serving base stations, resulting in better channel conditions and consequent larger bit rates. The evolutionary trend in this direction has been in the past the provision of services using hierarchical layers of macro, micro and pico cells, the latter for high traffic density areas, while it is envisaged in the short future the deployment of femto-cells or home base stations that has already started in some places. In fact, some predictions from 2006 in [1] envisaged 102 millions of femtocell users over 32 millions of access points worldwide for 2011.

- Increasing the total available bandwidth, i.e. having a larger amount of radio spectrum to deploy the services. In that respect, there has always been a continuous trend to increase the occupied spectrum. Starting from the $50 \mathrm{MHz}$ initially assigned to GSM in the band of $900 \mathrm{MHz}$, this was increased towards roughly $350 \mathrm{MHz}$ currently occupied by $2 \mathrm{G}$ and $3 \mathrm{G}$ systems in frequencies of up to $2.1 \mathrm{GHz}$. Following this expansion, and although there will be still some room for allocating new systems, such as in the case of LTE, the room for improvement here is quite limited, since there is less and less spectrum available in those frequency bands that would be more suitable for deploying a wireless network. To overcome this issue, this will claim for the adoption in the next decades of novel spectrum usage paradigms that enable a more efficient usage of the available spectrum.

In accordance with the above discussions, the natural evolution that one can envisage for the next decade in wireless communications networks resides on the principles of heterogeneity due to the coexistence of multiple technologies with different capabilities and cell ranges, on the need to have smart and efficient strategies to cope with the high demand of broadband services, and also on a longer term basis on the introduction of flexibility in the way how spectrum is being managed. These aspects are further discussed in the following points. 
Heterogeneity in networks and devices. Multiple technologies are being deployed one on top of the other, such as the cellular evolution GSM-UMTS-HSPA-LTE or the profusion of WLANs and short-range technologies (e.g. Bluetooth, RF-ID). Then, the future wireless arena is expected to be heterogeneous in nature, with a multiplicity of cellular, local area, metropolitan area and personal area technologies coexisting in the same geographical region. In addition even for a given cellular technology also different deployments will be envisaged particularly in urban areas, where macro, micro, pico and femtocell deployments will be coexisting as the means to achieve the desired large capacities. Heterogeneity will not only be present from the network perspective but also very different types of wireless devices will exist. In addition to classical phones, also PDAs, navigators, sensors or more simple RF-ID devices will constitute different ways of having access to the wireless services.

Network heterogeneity has been in fact regarded as a new challenge to offer services to the users over an efficient and ubiquitous radio access thanks to coordinating the available Radio Access Technologies (RATs), which on the other hand exhibit some degree of complementariness. In this way, not only the user can be served through the RAT that fits better to the terminal capabilities and service requirements, but also a more efficient use of the available radio resources can be achieved. This calls for the introduction of new RRM algorithms operating from a common perspective that take into account the overall amount of resources available in the existing RATs, and therefore are referred to as Common RRM (CRRM), Joint RRM (JRRM) or Multi RRM (MRRM) [2].

In this context, smart RAT selection and vertical handover mechanisms are able to achieve significant capacity gains in networks such as GSM/GPRS/UMTS in which performance obtained can be in a similar order of magnitude [3,4]. Nevertheless, when considering high bit rate networks such as HSPA and even more LTE or LTEAdvanced, the big difference in terms of performance when compared to e.g. GPRS or UMTS R99, one could expect that capacity improvements achieved thanks to CRRM are only marginal. In spite of this fact, even in this case the complementarities of the access networks can be used to cope with service and terminal differentiation, in the sense that legacy networks can still be used to provide those less bit rate demanding services (e.g. voice) and to provide service to those terminals not yet supporting new technologies. This would allow improving the peak bit rates attained by high demanding services at the new technologies such as LTE.

Multiplicity of data services with high bit rate requirements. We have already witnessed the explosion of wireless data services, mainly thanks to the deployment of wideband technologies (e.g. HSPA) that allow a user experience closer to the one that can be achieved in wired networks. In future years, it is expected that this trend continues and even that other services achieve more significant market-shares, such as mobile TV, multimedia, games, etc., and also some other specific services dealing with healthcare (e.g. through biosensors) or location-based services thanks to the proliferation of navigators.

The convergence between mobile and data access internet-based services posed specific challenges to wireless networks designers about how to exploit the set of 
resources available as efficiently as possible. In the first wireless networks, since the only application supported was voice, main requirements were to keep a good subjective voice quality and a bounded blocking probability to ensure accessibility in the coverage area. These two aspects were easily handled by means of an adequate network planning that ensured the SINR constraints in the coverage area and that a sufficient number of channels were available. However, with the evolution of wireless networks to support the provision of different types of services with different requirements (e.g. mixing both real time and non-real-time applications with different degrees of user profiles) and the development of more sophisticated and flexible radio access technologies (e.g. based on CDMA or OFDMA), QoS provision cannot be achieved only through a static planning procedure, but it is dynamically pursued by a set of functionalities grouped under the general term "Radio Resource Management" (RRM) [5,6].

RRM functions are responsible for taking decisions concerning the setting of different parameters influencing on the radio interface behaviour. This includes aspects such as the number of simultaneous users transmitting with their corresponding powers, transmission bit rates, the corresponding code sequences or sub-carriers assigned to them, the number of users that can be admitted in a given cell, etc. Since the different RRM functions will target to track different radio interface elements and effects, they can be classified according to the time scales they use to be activated and executed. In that sense, RRM functions such as power control, scheduling or link adaptation mechanisms tend to operate in short term time scales (typically in the order of milliseconds) while other functions such as admission control, congestion control or handover tend to operate in longer term time scales (typically in the order of seconds).

Among the different RRM strategies, and given the envisaged importance of data services in future networks, it is expected that smart and efficient scheduling mechanisms, aiming at the assignment of resources in the short term to the users requesting service, targeting the maximization of system usage and the fulfilment of QoS constraints, become particularly relevant in the next decade. This has already been the case of $3.5 \mathrm{G}$ technologies like HSPA, operating in packet switched mode, so that resources are not permanently assigned to users but they are only assigned when required, and will be the case of future LTE and LTE-Advanced systems.

Trend towards decentralisation and flat architectures. The architecture of the radio access networks has been traditionally hierarchical, composed by a number of base stations connected to some controller node (e.g. the BSC in 2G systems or the RNC in $3 \mathrm{G}$ ) that is in charge of all the management, decision and control functions related with the radio interface. As a result, RRM functions have been classically implemented in a centralized way, i.e. in central network nodes that can have a more complete picture of the radio access status than a particular node, so that decisions can be made with more inputs. However, centralized implementations have some drawbacks in terms of increased signaling load or transfer delay of the algorithm' s inputs to the central node, which prevents an efficient implementation of short-term RRM functions such as packet scheduling, resulting in larger latencies for 
the information delivered and in a lower capability for the scheduling algorithm to follow channel variations, with the corresponding decrease in spectrum efficiency.

While the above limitations could be acceptable in classical circuit-oriented networks and for low bit rate services, they can bring significant inefficiencies when broadband services are to be provided. This explains why already wireless cellular technology evolutions (e.g. HSPA) exhibit the trend towards implementing RRM functions on the radio access network edge nodes (e.g. base stations), which are in charge of executing fast packet scheduling mechanisms with the corresponding decrease in latencies. To a larger extent, this trend is also present in the SAE (System Architecture Evolution) for LTE, which tries to capture a flat architecture in which decision and control functionalities of the radio interface are moved towards the base stations, including additional capabilities to coordinate among them through direct interfaces (e.g. the X2 interface).

On the other hand when combining this trend with the expected proliferation of femto-cells in future years, which will in fact contribute to an important modification in the classical cellular approaches, it is clear that the degree of coordination among cells should necessarily be limited to relatively slow varying information, otherwise the required signalling to communicate multiple cells would prohibitively increase. The clear result of this will be that autonomous operation of base stations, including the necessary adaptive mechanisms to analyse their environment and take the appropriate configuration decisions, will be a must.

Going one step further in this decentralization direction leads to the implementation of distributed RRM functions at the terminal side, where relevant information for making smarter decisions is kept. This approach has claimed to be inefficient in the past because of the limited information available at the terminal side (e.g. the terminal does not know what is the cell load). Nevertheless, this can be overcome if the network is able to provide some information or guidelines to the terminal assisting its decisions, in addition to the valuable information that is already kept by the terminal (e.g. knowledge of the propagation conditions to surrounding cells). In this way, while a mobile-assisted centralized decision making process requires the inputs from many terminals to a single node, the network-assisted decentralized decision making process requires the input from a single node to the terminals, which can be significantly more efficient from a signalling point of view. An example of this trend is the IEEE P1900.4 protocol.

Flexible spectrum management. The regulatory perspective on how the spectrum should be allocated and utilized in a complex and composite technology scenario is evolving towards a cautious introduction of more flexibility in spectrum management together with economic considerations on spectrum trading. This new spectrum management paradigm is driven by the growing competition for spectrum and the requirement that the spectrum is used more efficiently [7]. Then, instead of the classical fixed spectrum allocation to licensed systems and services, which may become too rigid and inefficient, it is being recently considered the possibility to use Flexible Spectrum Management (FSM) strategies that dynamically assign spectrum bands in accordance with the specific traffic needs in each area [8]. There are differ- 
ent FSM scenarios presenting different characteristics in terms of technical, regulatory and business feasibility. While a fully enabled FSM scenario can be envisaged at a rather long-term perspective (e.g. maybe in more than 20 years), there are already some basic FSM scenarios that will likely become a reality in the short term. In particular, spectrum refarming, providing the possibility to set-up communication on a specific RAT in different frequency bands (e.g. refarming of GSM spectrum for UMTS/HSxPA communications), is a first example. Another case for FSM arises from the so-called digital dividend, which corresponds to the frequencies in the UHF band that will be cleared by the transition of analog to digital television. The cleared spectrum could be utilized by mobile TV or cellular technologies like UMTS, LTE, WiMAX, etc. and also for flexibly sharing spectrum between smart radio technologies. The exploitation of the so-called TV White Spaces, which refer to portions of spectrum that are unused either because there is currently no license holder for them, or because they are deliberately left unused as guard bands between the different TV channels, is another opportunity for FSM mechanisms.

Based on the above trend, a possible classification of spectrum usage models is given in [9] as follows:

- Exclusive Usage Rights model. This model consists in the assignment of exclusive licenses under which technology, services and business models are at the discretion of the licensee. In this case, harmful interference is avoided through technology-neutral restrictions. Licenses may be traded in an open market, reducing barriers to entry and encouraging an efficient use of the spectrum.

- Spectrum Commons model. This model promotes shared access to available spectrum for a number of users, so that in this case the networks themselves are responsible for ensuring that no interference exists. Under this model, two different levels exist. The first one is the public commons model, in which spectrum is open to anyone for access with equal rights, as it would be the case of e.g. current wireless standards in license-free ISM band. The second possibility is the so-called private commons model, in which the license holder enables multiple parties to access the spectrum in existing licensed bands, under specific access rules set by the primary license holder.

- Opportunistic spectrum access model. Under this model, secondary users are allowed to independently identify unused spectrum bands at a given time and place and utilize them while not generating harmful interference to primary license holders. Opportunistic spectrum access may take the form of either underlay access, in which signal powers below the noise floor (e.g. UWB) are used, or overlay access, which involves the detection and use of spectrum white spaces. In this case, cognitive radio networks become key in providing the awareness and adaptability required.

The concept of flexible spectrum management is closely related with the reconfigurability needed in radio equipment in order to cope with variations in the bands allocated to each system. Such reconfigurability can be achieved through the application of SDR technologies. In that respect, different initiatives have been started, such as the ETSI Reconfigurable Radio Systems (RRS) Technical Committee (TC). 
It carries out a feasibility study of the standardization activities related to Reconfigurable Radio Systems encompassing radio solutions related to Software Defined Radio (SDR) and Cognitive Radio (CR) research topics, trying to identify requirements from relevant stakeholders, such as manufacturers or operators. In particular, requirements and reference architectures for reconfigurable base stations and mobile devices have been identified, and also a functional architecture for the management and control of RRSs has been developed, including Dynamic Self-Organizing Planning and Management, Dynamic Spectrum Management and Joint Radio Resource Management functionalities [10]. Based on this it is expected for the next decade that reconfigurability will also play a relevant role in wireless communications networks.

\section{Self Organising Networks}

Third-generation (3G) mobile communication systems have seen widespread deployment around the world. The uptake in HSPA subscriptions, numbering well over 85 million at the start of 2009 [11] indicates that the global thirst for mobile data has just begun: broadband subscriptions are expected to reach 3.4 billion by 2014 and about 80 percent of these consumers will use mobile broadband.

Operators are doing business in an increasingly competitive environment, competing not only with other operators, but also with new players and new business models. In this context, any vision for technology evolution should encompass an overall cost-efficient end-to-end low latency proposition. By the end of 2004, 3GPP started discussions on $3 \mathrm{G}$ evolution towards a mobile communication system that could take the telecoms industry into the 2020s. Since then, the 3GPP has been working on two approaches for $3 \mathrm{G}$ evolution: LTE and HSPA+. The former is being designed without backward compatibility constraints, whereas the later will be able to add new technical features preserving WCDMA/HSPA investments.

The introduction of LTE and LTE-Advanced systems in the near future over current existing networks will bring a high degree of heterogeneity with many operators having several mobile network technology generations coexisting (GSM/GPRS, UMTS/HSPA, LTE/LTE-Advanced). This will require spending considerable effort in planning, configuring, optimising, and maintaining their networks, consuming significant operational expenditure (OPEX), because with the increasing complexity in the network deployments, network optimization will involve the tuning of a multiplicity of parameters that have a relevant impact over system performance. Traditionally, this tuning was done manually or semi-automatically during initial network deployment phase, leading to static parameter settings. However, being wireless networks inherently dynamic and very sensitive to traffic and interference variations, which is particularly more relevant with the CDMA and OFDMA-based radio interfaces of $3 \mathrm{G}$ and LTE systems, this approach can easily lead to inefficient operations, which will become more critical when broadband services are to be provided.

On the other hand, the envisaged high density of small sites, e.g. with the introduction of femto-cells, and the pressure to reduce costs clearly indicate that deploying 
and running networks needs to be more cost-effective. Consequently, it will be very important for operators that future wireless communication systems bring significant reductions of the manual effort in the deployment, configuration and optimisation activities. Based on this, besides the evolution in radio technologies and network architectures, there is also an evolution in the conception on how these networks should be operated and managed, towards the inclusion of automated Self-Organizing Network (SON) mechanisms to provide the networks with cognitive capabilities enabling its self-configuration without the need of human intervention.

The introduction of Self-Organising Networks (SON) functionalities, aiming to configure and optimize the network automatically, is seen as one of the promising areas for an operator to save operational expenditures and in fact this concept is already being introduced in the context of E-UTRAN standardisation, since it will be required to make the business case for LTE more attractive. It can be envisaged that in the next decades different networks will make use of this concept. Standardization efforts are demanded to define the necessary measurements, procedures and open interfaces to support better operability under multi vendor environment.

As a result of the above, SON has received a lot of attention in recent years in 3GPP [12] or in other initiatives such as the NGMN (Next Generation Mobile Networks) project [13], an initiative by a group of leading mobile operators to provide a vision of technology evolution beyond $3 \mathrm{G}$ for the competitive delivery of broadband wireless services to increase further end-customer benefits. It can be consequently envisioned that SON mechanisms will play relevant role in the mobile networks in the framework of the next decade.

A Self-organizing Network (SON) is defined in [14] as a communication network which supports self-x functionalities, enabling the automation of operational tasks, and thus minimizing human intervention. Self-organisation functionalities should be able not only to reduce the manual effort involved in network management, but also to enhance the performance of the wireless network. SON functionalities are classified in [14] in the following categories or tasks:

- Self-configuration. It is the process where newly deployed nodes are configured by automatic installation procedures to get the necessary basic configuration for system operation. This process is performed in pre-operational state where the RF interface is not commercially active. After the first initial configuration, the node shall have sufficient connectivity towards the network to obtain possible additional configuration parameters or software updates to get into full operation.

- Self-planning. It can be seen as a particular situation of self-configuration comprising the processes were radio planning parameters (such as neighbor cell relations, maximum power values, handover parameters, etc.) are assigned to a newly deployed network node.

- Self-Optimization. It is the process where different measurements taken by terminals and base stations are used to auto-tune the network targeting an optimal behavior by changing different operational parameters. This can provide quality and performance improvements, failure reductions and operational effort minimisation. This process is accomplished in the operational state where the RF interface is commercially active. 
- Self-managing. This corresponds to the automation of Operation and Maintenance $(\mathrm{O} \& \mathrm{M})$ tasks and workflows, i.e. shifting them from human operators to the mobile networks and their network elements. The burden of management would rest on a mobile network itself while human operators would only have to provide high level guidance to O\&M. The network and its elements can automatically take actions based on the available information and the knowledge about what is happening in the environment, while policies and objectives govern the network O\&M system.

- Self-healing. This process intends to automatically detect problems and to solve or mitigate them avoiding impact to the users. For each detected fault, appropriate alarms shall be generated by the faulty network entity. Self-healing functionality monitors the alarms, and when it finds alarms that could be solved automatically, it analyses more necessary correlated information (e.g. measurements, testing results, etc) to trigger the appropriate recovery actions to solve the fault automatically.

Operator use cases have been formulated for different stages in [15] such as planning (e.g. eNode B power settings), deployment (e.g. transport parameter set-up), optimization (e.g. radio parameter optimization that can be capacity, coverage or performance driven) and maintenance (e.g. software upgrade). Similarly, in [16] different use cases are identified addressing self-optimisation, self-healing and selfconfiguration functions. These use cases include self-optimisation of Home eNode $\mathrm{B}$, load balancing, interference coordination, packet scheduling, handover, admission and congestion control, coverage hole detection and compensation, cell outage management, management of relays and repeaters and automatic generation of default parameters for the insertion of network elements.

3GPP progress in the context of SON is being reflected e.g. in [17], where use cases are being developed together with required functionalities, evaluation criteria and expected results, impacted specifications and interfaces, etc. Similarly, ICT-FP7 SOCRATES project is developing in deeper detail different use cases, such as cell outage [18]. Paradigms related to SON are discussed in an early paper in this area [19]. Additionally, in [20] the self-configuration of newly added eNode-Bs in LTE networks is discussed and a self-optimisation load balancing mechanism is proposed as well.

Generally self-x functionalities will be based on a loop (self-x cycle) of gathering input data, processing these data and deriving optimized parameters. A generic SON procedure is illustrated in Fig. 1, where network inputs coming from different sources (e.g. network counters, measurement reports, etc.) can be considered in the form of Key Performance Indicators (KPIs). The procedure acts as a loop that continuously interacts with the real network based on observation and actions. At the observation stage, the optimisation procedure collects information from the different KPIs available. Then, the optimisation procedure will contain different processes for each of the optimisation targets specified based on operator policies (e.g. avoidance of coverage holes, interference reduction, reduction of overlapping between cells, etc.). For each optimisation target a procedure involving the processing the KPIs coming from the observation phase (e.g. combining several KPIs, obtaining 


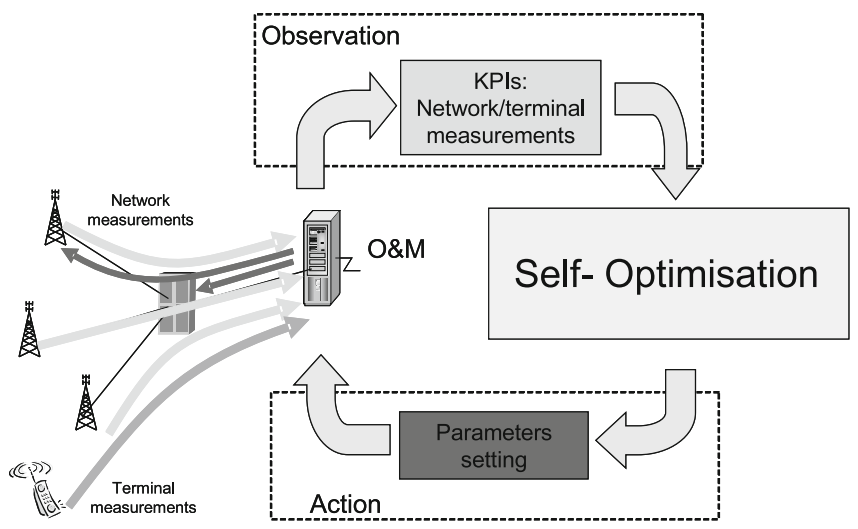

Fig. 1. Network optimisation loop

statistical measurements such as averages or percentiles, etc.), the detection of the sub-optimal operation based on some criteria dependent on the specific target, and the optimisation search to find the proper parameter setting to solve the sub-optimal operation. The final result of the optimisation will be the adequate configuration of selected network parameters (e.g. antenna downtilts, pilot powers, RRM parameters configuration, etc.) affecting either the cell where the sub-optimal operation has been found and/or its neighbouring cells. This change in the configuration parameter(s) turns out to be the action executed over the network.

Clearly, the SON concept at the largest extent of a totally automatic network, able to operate with minimum human intervention, is quite ambitious and challenging, so that it can be anticipated that SON will continue as a hot research topic in coming years requiring further research efforts to facilitate its practical implementation. In particular, some of the aspects that must be properly covered include: (1) to find an appropriate trade-off between performance gains and implementation complexity in terms of signalling, computational requirements and measurements, (2) ensure the convergence of the algorithms towards stable solutions within a given timing requirement and (3) ensure the robustness of the mechanisms in front of missing, wrong or corrupted input measurements.

The above context outlines a roadmap for the next decade from current deployed networks (2G, 3G HSPA), mainly managed by centralized remote operations and maintenance $(\mathrm{O} \& \mathrm{M})$ applications with intensive human intervention, to future SONs (LTE, HSPA+). Targeting a fully SON as an ultimate objective, an evolutionary process can be envisaged, with the progressive inclusion of the SON culture [21]. Given that changing the network configuration/parameterisation is a critical action from the operator's perspective, automated optimisation procedures are likely to be introduced in a step-by-step approach, starting with automatic sub-optimal behaviour detection mechanisms and establishing the corrective actions to overcome them through the semi-automated optimisation of the different parameters, perhaps with some human intervention. An ultimate view, where a joint self-optimisation 
of several targets is performed will still require a lot of research efforts, due to the complex interactions between these targets. Supporting tools and/or models for this stage will also be needed to estimate the impact of a given parameter change on the live network. In accordance with this envisaged evolutionary approach, in [21], a roadmap is presented starting from the practical application of UMTS optimisation towards automated self-optimisation procedures in LTE.

While SON concepts can be regarded as a relatively short term vision for the next decade, going one step further brings the idea of automated network operation towards a more complete vision envisaging end-to-end goals. This can be framed under what has been coined under the term "cognitive network". In fact, the word 'cognitive' has recently been used in different contexts related to computing, communications and networking technologies, including the cognitive radio terminology coined by. J. Mitola III [22] and the cognitive network concept. Several definitions of cognitive networks have been proposed up to date, but all of them have one thing in common: they present a vision of a network which has the ability to think, learn and remember in order to adapt in response to conditions or events based on the reasoning and prior acquired knowledge, with the objective of achieving some end-to-end goals [23].

The central mechanism of the cognitive network is the cognitive process, which can perceive current network conditions, and then plan, decide and act on those conditions. Then, this is the process that does the learning and decides on the appropriate response to observed network behaviour [24]. The cognitive process acts following a feedback loop in which past interactions with the environment guide current and future interactions. This is commonly called the OODA (Observe, Orient, Decide and Act) loop, which has been used in very different applications ranging from business management to artificial intelligence, and which is analogous to the cognition cycle described by Mitola in the context of cognitive radios.

In the cognitive behaviour, the perception of the stimulus is the means to react to the environment and to learn the rules that permit to adapt to this environment. This is directly related with the observation phase of the cognition cycle, which tries to capture the network status. This involves a large number of measurements and metrics that can be obtained at different network elements. It is important to identify which are the most relevant ones for each phase of the cognition cycle, since measurements relevant for a particular function need to reach the network element where the corresponding function is implemented. Measurements and metrics of interest may be at connection level (e.g. path loss from terminal to cell site, average bit rate achieved over a certain period of time, etc.) or at system level (e.g. cell load, average cell throughput achieved over a certain period of time, etc.). It is worth mentioning here that the observation function may bring the "sensing concept" to all the layers of the protocol stack, depending on which are the aspects to be observed (e.g. not only the measurement of radio electrical signals at the physical layer should be addressed but also the configuration of different applications or network protocols can be also considered). Any means that permits to analyze the environment, and that may be helpful for the adaptation of the communication system to the constraints imposed by the environment, is worth being taken into account. 
Being the learning one the most relevant aspects for a cognitive network, many strategies have been envisaged in the literature as learning procedures with the ultimate goal of acquiring knowledge. In particular, machine learning has been widely considered as a particularly suited framework, with multiple possible approaches. The choice of the proper machine learning algorithm depends on the desired network goals. In any case, one of the main challenges here is that the process needs to be able to learn or converge to a solution faster than the network status changes, and then re-learn and re-converge to new solutions when the status changes again, so that convergence issues are of particular importance. Machine learning algorithms can also be organized in different categories (supervised learning, unsupervised learning, semi-supervised learning, and reinforcement learning being the most common ones) depending on how their desired outcome is obtained. Some of the identified machine learning strategies studied in the literature are neural networks, genetic algorithms, expert systems, case-based reasoning, Bayesian statistics, Q-learning, etc.

\section{Opportunistic networks}

Opportunistic networking primarily stems from mobile ad hoc networking, that is, the research area that applies to scenarios where the complete absence of any supporting infrastructure is assumed. Nowadays there is an ongoing intense work on this topic, however only a few killer applications have been envisaged so far. Opportunistic networks will spontaneously emerge when users carrying mobile devices, such as PDA, laptops, mobile phones, etc., meet. According to the opportunistic networking paradigm, partitions, network disconnections and nodes mobility are more regarded as challenging chances rather than limiting factors or exceptions. Mobility, for example, is considered as a way to connect disconnected network portions; partitions or network disconnections are not regarded as limitations since, in a longer temporal scale and by exploiting a store-and-forward approach, the information will finally flow from a source to the final destination. So in opportunistic networks, delivering can be considered just a matter of time. Opportunistic networking shares also many aspects with delay-tolerant networking, which is a paradigm exploiting occasional communication opportunities between moving devices. These occasional inter-contacts can be either scheduled or random, although conventional delay-tolerant networks (DTNs) typically consider the communication opportunities as scheduled in time. Opportunistic networking and delay tolerant networking have been somehow distinguished so far in the literature, although a clear classification does not exist. However, as a common understanding, opportunistic networking could be regarded as a generalization of delay-tolerant networking with no a-priori consideration of possible network disconnections or partitions. This basically will result in a different routing mechanisms being employed by the two networking paradigms: DTNs will exploit the same Internet routing approach by only taking into account the possibility to have long delays; on the contrary, in opportunistic networks paths will be dynamically built so as to overcome possible disconnections met along the path. 
In the next years we expect diffusion of numerous opportunistic networking scenarios where individuals, equipped with very powerful mobile communication devices, like smart phones or PDAs, will establish transparent direct communication links to each other. The technology for supporting such a kind of immersive communications is already available and it is predictable that, although wireless enhanced communications between devices will not replace standard communications such as SMS or phone calls, they will keep increasing their weight in next generation scenarios.

These enhanced networks formed by millions of mobile devices carried out by moving users are expected to increase in size becoming very crowded, especially in case of metropolitan areas. These networks, according to the traditional opportunistic perspective, will be mainly characterized by sparse topology, lack of full connectivity and sporadic communication opportunities. Overlapping between these network communities will be envisaged as the possibility to exploit superposition to strengthen and enhance the security of communication among network members. To this purpose the concept of community profile and/or homophily can be employed. The community profile will be used to identify the overlapping communities a user belongs to, and the strength of the logical relationships that a user has with other community users. On the other hand, homophily can help to characterize the degree of similarities between users of different communities and the degree of trust between them. This trust could be used in selecting possible forwarder nodes for information according to the opportunistic networking paradigm.

In this context, exploiting the so-called "social-awareness" (i.e., information about the social relationships and interactions among the members) has been recently proposed to perform data forwarding in opportunistic networks. To this purpose, some solutions have been envisaged, which use information on how users join together in social communities, to create efficient content replication schemes in opportunistic networks [25].

Another interesting application envisaged for wireless opportunistic scenarios is related to delay tolerant data broadcasting [26]. More in depth, due to the high density of mobile devices which are expected to be available in short time, these nodes could be exploited as data broadcaster, for example as an alternative to terrestrial and satellite broadcasting systems. This would represent an un-licensed public broadcasting tool alternative to those traditionally used which are also limited by frequency spectrum licence availability and concession rights, as well as censorship or political constraints. Many channels will be available and groups of users willing to share a special type of contents, e.g. music contents, will use the same channel. Epidemic and more controlled approaches for data forwarding will be supported. To allow this contents' dissemination, dock places will be available where contents could be downloaded from the Internet. Podcasting could for example represent an interesting tool for such a distribution of contents. Podcasting [27] is typically thought for downloading contents to a mobile media player. These contents can then be played back on the move. However, the main limitation with the current idea of podcasting is the impossibility of separating move and downloading of contents from a dock media station. This obviously poses limits in terms of use of the service since there could be 
hours between downloading opportunities. To improve this aspect and broaden the idea of podcasting, the high penetration and density of short-range wireless communications could be exploited. In fact, iPods, Zunes, or smart phones could represent a vehicle of epidemic multihop resource distribution, without relying on any infrastructure support. This podcasting service would allow the distribution of contents using opportunistic contacts whenever podcasting devices are in wireless communication range without any limitation of proximity to dock stations. Such epidemic dissemination approach is expected to be very successful and penetrating especially in urban areas where people meet in office, elevators, public transportations, sport areas, theatres, music events, etc.

All these elements will also contribute to the realization of a Minority Report-like communication scenario where information about user profiles and preferences will be stored by the environment at servers and used to foster service personalization and commercial advertisement based on node position and/or habits and requirements. This will obviously open new perspectives for personalized advertisement and marketing which will clearly pass through considerations of user preferences and habits being published through a context profile stored at the dock stations.

Opportunistic networking will be exploited also in the view of distributing contents retrieved by an integrated set of sensors deployed in devices carried by users moving around an interest area. Academic Research Projects proposing similar ideas have recently appeared (e.g. Cenceme [28], Metrosense [29], CRAWDAD [30], etc.) but it is envisaged that in a more large scale sensor network, these contents could be distributed around and infostations used to collect them. This would allow to both derive some detailed and realistic user mobility models, and to characterize variations in interest parameters (for example temperature or humidity) on a metropolitan area scale, as well as to study the statistics of interactions among moving users, even to use them as feedback for studying evolution of diseases propagation, thus trying to prevent them. Also this information about profiles is thought to be integrated into the virtual world of Second Life-like environments.

Finally, deep space communications are another significant application scenario especially for delay tolerant and, thus, opportunistic networking too. In fact, in case of satellite communications and/or communications between the international space station and users on the Earth or artificial satellites in the space, incredibly high delay should be considered. This impacts on the possibility to exchange data since often satellites are in visibility only for few minutes every week/month and this short time should be used as much efficiently as possible for transferring data in spite of the high delay (many minutes) and very high bit error rate. In the next years it is foreseeable that deep space communications will have a significant boost. This is because many international space projects have been launched by NASA, ASI, and others in the view of supporting, in 20-30 years, migration of Earth inhabitants to planets and satellites or international space stations. In this case, reliable communications between these people and those on the Earth should be granted and, consequently, opportunistic and delay tolerant networking become a promising and crucial tool to be exploited in such a science-fiction scenario. 


\section{Vehicular communication networks}

Vehicular networks, enabling the wireless communication between different cars and vehicles in a transportation system have been another topic that has received attention during the last years, driven mainly by the automotive industry and also by public transport authorities, pursuing the increase of both safety and efficiency in transportation means. Although a lot of effort has been devoted, no solutions are already available to the mass markets allowing the automatic operation of cars and the communications among them. It is thus expected that the evolution of wireless communications in different aspects, such as adhoc and sensor networks, distributed systems, combined operation of infrastructure and infrastructureless networks, etc. can become an important step so that vehicular communications become a reality in the next decades.

From a more general perspective, the term Intelligent Transportation Systems (ITS) has been used referring to the inclusion of communication technologies to transport infrastructure and vehicles targeting a better efficiency and safety of road transport. Vehicular Networks, also known as Vehicular Ad-hoc NETworks (VANETs), are one of the key elements of ITS enabling the one hand the intervehicle communication and on the other hand the communication of vehicles with roadside base station. This communication is intended to exchange different types of messages, such as safety warnings or traffic information, which can help in avoiding both accidents and traffic congestions through appropriate cooperation.

The provision of timely information to drivers and concerned authorities should contribute to safer and more efficient roads, and in fact safety has been the main motivation for the promotion of vehicular networks. It is mentioned in [31] that if preventive measures are not taken, road death is likely to become the third-leading cause of death in 2020. On the other hand, most of the road accidents are associated with specific operations such as roadway departures or collisions at intersections that could be easily avoided by deploying adequate communication mechanisms between vehicles in which they could warn in advance other vehicles about their intentions of departing or arrivals at intersections.

Different initiatives have been launched in the last years in the area of vehicular communications and ITS, involving governmental agencies, car manufacturers and communication corporations. In the United States, the U.S. Department of Transportation launched the Vehicle Infrastructure Integration (VII) and the IntelliDrive programs targeting vehicle to vehicle and vehicle to roadside stations communications through Dedicated Short Range Communications (DSRC) [32,33]. In the context of ETSI, the Technical Committee on Intelligent Transport Systems (TC ITS) targets the creation and maintenance of standards and specifications for the use of information and communications technologies in future transport systems in Europe [34]. Most of the technical committee's ongoing standardization activities are focused on wireless communications for vehicle-to-vehicle and vehicle-to-roadside communications, with the goal to address the reduction of road deaths and injuries and to increase the traffic efficiency for transport systems with reduction of transport time, contributing to decrease polluting emissions. 
Different applications can be envisaged in the context of vehicular communication networks, ranging from safety aspects to a fully automated control of the car that could potentially be driven without human intervention. From the safety point of view, they can be used by vehicles to communicate imminent dangers to others (e.g. detecting an obstacle in the road, informing of an accident) or to prevent others from specific movements such as entering intersections, departing highways, lane changes, etc. and also the communications can help in automatically keeping a safe distance between a vehicle and the car ahead of it, etc. Another application could be related with traffic management, by providing traffic authorities with proper monitoring statistics that could lead to changes in traffic rules such as variable speed limits, adaptation of traffic lights, etc., in order to increase the fluency of traffic in a given road. Similarly, vehicular communications can also assist drivers providing information to facilitate the control of the vehicle, e.g. lane keeping assistance, parking, cruise control, forward/backward collision warnings, providing travel-related information such as maps or gas station locations, etc. Other applications could also be related with enabling faster toll or parking payments or with road surveillance mechanisms [35]. Finally, also private data communications, enabling e.g. Internet access, games, etc. to passengers inside the car can also be envisaged as an application of future vehicular communications.

From a standardisation point of view, the $5.8-\mathrm{GHz}$ frequency band was allocated by CEPT/ECC for vehicular communications, and an ITS European Profile Standard is being developed based on IEEE 802.11 and constitutes the basis for developing specifications on interoperability and conformance testings [36].

The IEEE 802.11p system, usually referred to as Wireless Access in Vehicular Environments (WAVE) [37], adapts the IEEE 802.11a standard used in WLANs to the vehicular environment, by addressing the specific needs of vehicular networks. Similarly IEEE 1609.X family of standards for WAVE defines the architecture, communications model, management structure, security mechanisms and physical access for high speed (i.e. up to $27 \mathrm{Mbit} / \mathrm{s}$ ) short range (i.e. up to $1000 \mathrm{~m}$ ) low latency wireless communications in the vehicular environment [38]. They deal with the management and security of the networks, addressing resource management and network and application layer aspects. In these standards, vehicular networks should be composed by three types of nodes. The first two are vehicles and roadside stations, including, respectively, vehicle On-Board Units (OBU) and RoadSide Units (RSU), the latter being some equivalent to $\mathrm{WiFi}$ access points to provide communication with the infrastructure. In addition, a third type of node is the Public Safety On Board Unit (PSOBU) which is a RSU with special capabilities used mainly in special cars such as police, ambulances or fire trucks in emergency situations. The network should support both private data communications (e.g. Internet connectivity on the move) and public communications, with a higher priority for the latter, since they include safety aspects. Although 802.11p and 1609 draft standards specify baselines for developing vehicular networks, many issues are not addressed yet and more research is required.

A widespread adoption of wireless vehicular communication technologies will require efficient use of the radio channel resources, due to the decentralized nature 
of the networks and the strict quality of service requirements of applications involving traffic safety issues. At the same time, the network infrastructure needs to be designed and implemented to provide reliable network access to objects travelling at high speeds. This will involve specific RRM mechanisms adapted to the characteristics of vehicular networks $[39,40]$.

\section{Future Internet and the Internet of Things}

Internet, and the possibility to connect any computer with any other around the world constituted in the last decades one of the major cornerstones of telecommunications, turning into one of these revolutionary changes that highly influenced the life and working style of people. Clearly, in this Internet revolution, also wireless technologies played a major role, enabling the connectivity anywhere and anytime without the need of having wired connections. Under this context, the evolution of Internet for the next decade has been coined under the term Future Internet, embracing new (r)evolutionary trends in terms of e.g. security, connectivity and context-aware applications, in which again also wireless technologies will become an important and relevant element for the success of the different initiatives.

In particular, one of the envisaged challenging goals for Future Internet is the possibility to interconnect not only people through computer machines (i.e. people connected everywhere and anytime) but also all type of inanimated objects in a communication network (i.e. connecting everything with everything), constituting what has been coined as the Internet of Things [41]. It presents a vision in which the use of electronic tags and sensors will serve to extend the communication and monitoring potential of the network of networks. This concept envisages a world in which billions of quotidian objects will report their location, identity, and history over wireless connections, so in order to make it true, this will require dramatic changes in systems, architectures, and communications [42,43].

Different wireless technologies and research disciplines can be embraced under the Internet of Things concept. Although Radio Frequency IDentification (RFID) and short-range wireless communications technologies have already laid the foundation for this concept, further research and development is needed to enable such a pervasive networking world, with the necessary levels of flexibility, adaptivity and security. Such technologies are needed as they provide a cost-effective way of object identification and tracking, which becomes crucial when trying to connect the envisaged huge amounts of devices. Also real-time localization technologies and sensor/actuator networks will become relevant elements of this new vision, since they can be used to detect changes in the physical status of the different things and in their environment. The different things will be enabled with the necessary artificial intelligence mechanisms allowing them to interact with their environment, detecting changes and processing the information to even take appropriate reconfiguration decisions. Similarly, advances in nanotechnology enabling the manipulation of matter at the molecular level, so that smaller and smaller things will have the ability to connect and interact, will also serve to further accelerate these developments. 
Obviously, in order to allow things to communicate with each other we need to address them. The problem is defining the most appropriate addressing schemes taking into account the existing standards. In fact, communications in wireless sensor networks will comply with the IEEE 802.15.4 standard, whereas, RFID communications are regulated by the EPCglobal standards. Recently, 6LowPan has been proposed by IETF to support IPv6 over IEEE 802.15.4 communication devices. In fact, IPv6 uses a 128 bit IP address which is large enough to accommodate all imaginable communication devices (consider that IPv4 addresses are almost exhausted). We may think to use IPv6 addresses for RFID tags as well. This will obviously require solutions for the mapping between IPv6 addresses and EPCglobal tag identifiers.

Wireless technologies under the Internet of Things concept will not only have to deal with short range communications but also it can be expected that wide area technologies (e.g. cellular) will also play an important role, since they will provide the different things with the ability to communicate at longer distances. This could be useful e.g. when a user could try to configure remotely through its mobile phone the adequate settings of electronic equipment at home. Appropriate traffic characterisation of these new applications would also be needed in order to adequately perform the network deployment.

Based on all the above, one can envisage for the next decades a new Internet concept, in which humans will be surrounded by a fully interactive and responsive network environment, constituted by smart things able to take appropriate decisions in accordance with environment variations and human needs, contributing to increasing the quality of life. Multiple applications can be envisaged, such as the intelligent electronic control of different machines and gadgets at home or in-car, able to configure and personalise in accordance to used preferences aspects as temperature, humidity, illumination, etc. Similarly, it could be possible to detect possible misbehaviours or failures using diagnostic tools in order to anticipate when human intervention is needed. Another field of applications where this could have applicability would be the e-health services or the support to the elderly and handicapped people in domestic tasks. However, the application scenario in which the Internet of Things concept can give the most important gains in terms of cost reduction and optimization is related logistics (logistics in the production process or in the transportation system). In fact, the possibility of keeping under control the position of each single item can really make the difference in the management decisions and, indeed, most of industrial interest into the Internet of Things is focusing on the applications in the logistic fields.

On the other hand, it is obvious that such a pervasive computing and communication infrastructure envisioned by the Internet of Things raises several concerns regarding privacy. In fact, a smart environment can collect a large amount of personal information that can be used to support added-value context aware services, but also to control people. The lots of talking and complaining about the announcement by an Italian retailer to tag their clothes with lifelasting RFID, demonstrates that people are not available to accept such technologies when they become a serious menace for their privacy. Accordingly, the Internet of Things will include several functionalities protecting personal information and ensuring that it will be used only for the purposes authorized by the information owner. 
Finally, we can gain a completely different perspective if we look at the Internet of Things along with information-centric networking, which is expected to be one of the other major components of the Future Internet. The key rationale behind information centric networking is that users look at the Internet for the information it provides and are not interested in the specific host that stores this information. Unfortunately, IPbased Internet has been developed around the host-to-host communication paradigm, which does not fit this novel idea of the Internet.

It is useful to analyze how the Internet of Things can coexist and even exploit information-centric networking in the Future Internet. The first attempt in this direction is based on the concept that each resource is described by a metadata called Versatile Digital Item (VDI), decoupled by the resource and usable to represent real world objects. VDIs for real world objects contain information about the current state of the thing, for example, current owner, current usage type, position, etc. This approach has two important advantages:

- position of VDIs does not necessarily change as things move. This simplifies mobility management significantly;

- VDIs can implement functionalities which, accordingly, will run in infrastructure nodes instead of the thing objects they describe. This is important as processing capabilities available in actual things are extremely limited.

The major issues related to such an approach regard the naming scheme to be utilized and the related routing protocol. In general, in information centric networking contexts we can distinguish two approaches for what concerns the naming strategy: flat and hierarchical naming.

Usually, flat naming schemes are utilized along with rendezvous node based schemes, in which the name is mapped to a node where the state information of the thing is stored through a well known hash function. This rendezvous node will receive all queries and all updates regarding the VDI. Routers will route the query to the rendezvous node by appropriate processing of such a name. However, flat names are not mnemonic and therefore, appropriate services/applications are needed to remember such names, which introduces further complexity in the system. Furthermore, it is difficult (or even impossible) to control the selection of the rendezvous node.

A different approach that exploits hierarchical naming in information centric networks has been proposed in [44]. In this case, routers maintain updated routing tables in which the entry key is not the IP address but the name of the resource. Using this approach, the routing tables increase with the number of resources introduced in the network. In a global Internet of Things, this would be a manifest weakness. However, observe that the weakness may be overcome by using a structured naming that allows keeping the routing table size as small as possible. 


\section{Extending networking concepts to nanoscale communications}

When trying to extrapolate networking problems to the future decades, with a long term horizon in mind, one of the emerging fields that is gaining momentum in the last years is the evolution of nano-technologies towards the "nanonetwork" concept, driven by the inspiration of applying biological systems and processes to networking technologies as it has been used in other fields such as optimisation, evolutionary and adaptive systems (e.g. genetic algorithms, ant colonies, etc.).

The term "nanotechnology" was first defined in [45] as the processing of, separation, consolidation, and deformation of materials by one atom or by one molecule, and this basic idea was further explored in more depth in the 1980s, with an acceleration of the activity in the field in the early 2000s. Nanotechnology enables the miniaturization and fabrication of devices in a scale ranging from 1 to 100 nanometers, considering a nano-machine as the most basic functional unit. Nano-machines are tiny components consisting of an arranged set of molecules which are able to perform very simple computation, sensing and/or actuation tasks [46]. Nano-machines can be further used as building blocks for the development of more complex systems such as nano-robots and computing devices such as nano-processors, nano-memory or nano-clocks.

In [47], the term "nanonetworks" is used to refer to the interconnection of nanomachines based on molecular communication. In general terms, a nano-machine is defined as "a device, consisting of nano-scale components, able to perform a specific task at nano-level, such as communicating, computing, data storing, sensing and/or actuation". The tasks performed by one nano-machine are very simple and restricted to its close environment due to its low complexity and small size. Then, the formation of a nanonetwork allows the execution of collaborative tasks, thus expanding the capabilities and applications of single nano-machines. For example, in case of nano-machines such as chemical sensors, nano-valves, nano-switches, or molecular elevators, the exchange of information and commands between them will allow them to work in a cooperative and synchronous manner to perform more complex tasks such as in-body drug delivery or disease treatments. Similarly, nanonetworks will allow extending the limited workspace of a single nano-machine enabling the interaction with remote nano-machines by means of broadcasting and multihop communication mechanisms.

Nanonetworks offer a wide range of application domains from nano-robotics to future health-care systems enabling the possibility to build from nano-scale body area networks to nano-scale molecular computers. Potential applications of nanonetworks are classified in [47] in four groups: biomedical, environmental, industrial and military applications, although they could also be used in other fields such as consumer electronics, since nanotechnologies have a key role in the manufacturing process of several devices.

Due to the small size of nano-machines that may be composed of just several moles of atoms or molecules in the orders of a few nanometers, traditional wireless communication with radio waves cannot be used for the communication. As a result, communication in nanonetworks is mainly to be realized through molecular 
communication means, making use of molecules as messages between transmitters and receivers. Two different and complementary coding techniques are explained in [47] to represent the information in nanonetworks. The first one, similar to what is done by traditional networks, uses temporal sequences to encode the information, such as the temporal concentration of specific molecules in the medium. According to the level of the concentration, i.e., the number of molecules per volume, the receiver decodes the received message, in a similar way as how the Central Nervous System propagates the neural impulses. The second technique is called molecular encoding and uses internal parameters of the molecules to encode the information such as the chemical structure, relative positioning of molecular elements or polarization. In this case, the receiver must be able to detect these specific molecules to decode the information. This technique is similar to the use of encrypted packets in communication networks, in which only the intended receiver is capable to read the information. In nature, molecular encoding is used in pheromonal communication, where only members of the transmitter specie can decode the transmitted message.

As noted above, nanonetworks cannot be considered a simple extension of traditional communication networks at the nano-scale, but they are a completely new communication paradigm, in which most of the communication processes are inspired by biological systems found in nature. A part from the differences in how the information is encoded, also the communication medium and the channel characteristics are different from those used in classical networks. Propagation speed differs from traditional communication networks, given that the information contained in molecules has to be physically transported from the transmitter to the receiver, and also propagation can be affected by random diffusion processes and environmental conditions, resulting in a much lower propagation speed. With respect to the transmitted information, as a difference from traditional communication networks, where information typically corresponds to text, voice or video, in nanonetworks, being the message a molecule, information is more related to phenomena, chemical states and processes. Correspondingly, most of the processes associated to nanonetworks are chemically driven, resulting in low power consumption.

As a result of the above considerations, most of the knowledge from existing communication networks is not suitable for nanonetworks, thus requiring innovative networking solutions according to the characteristics of the network components and the molecular communication processes. While some research efforts have been already carried out, many open research issues remain to be addressed for a practical realization of nanonetworks [48]. Firstly, further exploration of biological systems, communications and processes, should allow the identification of efficient and practical communication techniques to be exploited. Furthermore, it is to be studied the possible applicability of the definitions, performance metrics and basic techniques of classical networks, such as multiplexing, access, routing, error control, congestion, etc. With respect to the molecular communication, there are many open questions regarding the transmission and reception such as how to acquire new molecules and modify them to encode the information, how to manage the received molecules, and how to control the binding processes to release the information molecules to the medium. Similarly, communication reliability is another key issue in nanonetworks, 
since some applications will require reliable mechanisms to monitor or interrupt selective transmissions or on-going processes, while at the same time some molecular communication schemes are subject to random processes preventing to guarantee correct reception of transmitted messages. Consequently, communication reliability should be investigated by considering random communication features and particular infrastructures in molecular communication. Once the basic nanonetwork components are built, the transmission is controlled and the propagation is understood, advanced networking knowledge can be applied to design and realize more complex nanonetworks, even using layered architectures including medium access protocols, routing schemes and application interfaces [47].

As stated in [48], different studies have been performed on the design of nanoscale communication, introducing design approaches for molecular communication $[46,49,50]$. Other studies address the single molecular communication channel from an information theoretical perspective [51] and an adaptive error compensation mechanism for improving molecular communication channel capacity [52]. Molecular multiple-access, relay and broadcast channels are modelled and analyzed in terms of capacity limits in [53]. Although the first theoretical studies on nanonetworks are already there, there is still a lot of work to do before this promising field can become a reality. An interdisciplinary scientific approach involving networking concepts and biological aspects is needed to address the identified research challenges.

\section{Conclusions}

This section addresses heterogeneous and opportunistic wireless networks, providing some views on the possible evolution of future wireless networks, on some of the envisaged elements that will guide this evolution, and on the design challenges that are currently at stake.

The implications of network heterogeneity on how wireless networks will be operated and perceived by the users are presented, with the corresponding requirements for smart radio resource management strategies. The basic ingredients allowing to successfully coping with the increasing demand of traffic and bit rate are addressed: increase of the spectral efficiency, i.e., the number of bits/s that can be delivered per unit of spectrum; increase of the number of base stations to provide a service in a given area; and increasing the total available bandwidth. Within this area, the heterogeneity in networks and devices is also addressed, together with the multiplicity of data services with high bit rate requirements. Trends towards decentralisation and flat architectures, and perspectives on flexible spectrum management are shown as well.

Self-organising networks are included in the analysis, since it is expected that many mechanisms will be established to allow the self-configuration of a network with minimum human intervention. The features to be included in such an approach include: self-configuration, self-planning, self-optimisation, self-managing, and self-healing. 
Opportunistic networks are also addressed, linking with delay-tolerant networks (among others), vehicular communication networks are analysed, linking with Intelligent Transportation Systems, and the Future Internet is viewed from the Internet of Things and RFID perspectives. Finally, a long term perspective is given towards the futuristic concept of the nanonetworks that target the extension of networking concepts towards the nano-scale communications.

\section{References}

1. ABI Research. Femtocell access points: Fixed-mobile convergence for residential, smb, and enterprise markets. Available: http://www.abiresearch.com (2006).

2. Tölli A., Hakalin P., Holma H.: Performance evaluation of common radio resource management (crrm). In: Proc. IEEE Int. Conf. Commun., New York, NY, pp. 3429-3433 (2002).

3. Pérez-Romero J., Sallent O., Agustí R.: On the optimum traffic allocation in heterogeneous CDMA/TDMA networks. IEEE Trans. Wireless Commun. 6(9), pp. 3170-3174 (2007).

4. Pérez-Romero J., Sallent O., Agustí R., Díaz-Guerra M.A.: Radio resource management strategies in UMTS. Wiley \& Sons, New York, NY (2005).

5. Zander J.: Radio resource management in future wireless networks: requirements and limitations. IEEE Commun. Mag. 35(8), pp. 30-36 (1997).

6. Sallent O., Pérez-Romero J., Agustí R., Casadevall F.: Provisioning multimedia wireless networks for better QoS: RRM strategies for 3G WCDMA. IEEE Commun. Mag. 41(2), pp. 100106 (2003).

7. Hoffmeyer J.A.: Regulatory and standardization aspects of dsa technologies - global requirements and perspectives. In: Proc. IEEE Int. Symp. New Frontiers in Dynamic Spectrum Access Networks, Baltimore, MD (2005).

8. Buddhikot M., Ryan K.: Spectrum management in coordinated dynamic spectrum access based cellular networks. In: Proc. IEEE Int. Symp. New Frontiers in Dynamic Spectrum Access Networks, Baltimore, MD (2005).

9. Xiao F.H.Y (Ed.): Cognitive Radio Networks. CRC Press, Boca Raton, FL (2009).

10. Mueck M., et al.: ETSI reconfigurable radio systems: Status and future directions on software defined radio and cognitive radio standards. IEEE Commun. Mag. 48(9), pp. 78-86 (2010).

11. UMTS Forum: Annual Report 2008 and Directions for 2009 (2009).

12. 3GPP Telecommunication Management: Self-Organizing Networks (SON), Concepts and Requirements (Release 8). Tech. Rep. (2008).

13. Next Generation Mobile Networks (NGMN): NGMN Recommendation on SON and O\&M Requirements (2008).

14. Bogenfeld E., Gaspard I. (Eds.): Self-x in Radio Access Networks (2008).

15. Next Generation Mobile Networks (NGMN): NGMN Use Cases related to Self Organising Network, Overall Description (2007).

16. Schmelz L., der Berg J.V., Litjens R., Zetterberg K., Amirijoo M., Spaey K., Balan I., Scully N., Stefanski S.: Self-organisation in wireless networks - use cases and their interrelation. In: Proc. WWRF Meeting, Paris, France (2009).

17. 3GPP Telecommunication Management: Evolved Universal Terrestrial Radio Access Network (E-UTRAN). Self-configuring and self-optimizingnetwork use cases and solutions. Tech. Rep. (2010).

18. Amirijoo M., Jorguseski L., Kurner T., Litjens R., Neuland M., Schmelz L., Turke U.: Cell outage management in LTE networks.

19. Prehofer C., Bettstetter C.: Self-organization in communication networks: Principles and design paradigms. IEEE Commun. Mag. 43(7), pp. 78-85 (2005).

20. Hu H., Zheng X., Yang Y.: Self-configuration and self-optimization for LTE networks. IEEE Commun. Mag. 48(2), pp. 94-100 (2010). 
21. Sallent O., Pérez-Romero J., Sánchez-González J., Agustí R., Díaz-Guerra M.A., Henche D., Paul D.: A roadmap from UMTS optimisation to LTE self-optimisation, to appear.

22. Mitola, J. III, Maguire G.: Cognitive radio: Making software radios more personal. IEEE Personal Commun. 6(4), pp. 13-18 (1999).

23. Mahmoud Q. (Ed.): Cognitive Networks: Towards Self-Aware Networks. Wiley and Sons. New York, NY (2007).

24. Thomas R., DaSilva L., MacKenzie A.: Cognitive networks. In: Proc. IEEE Int. Symp. New Frontiers in Dynamic Spectrum Access Networks, Baltimore, MD (2005).

25. Pantazopoulos P., Stavrakakis I., Passarella A., Conti M.: Efficient social-aware content placement for opportunistic networks. In: Proc. Int. Conf. Wireless On-demand Network Systems and Services, Kranjska Gora, Slovenia (2010).

26. Karlsson G., Lenders V., May M.: Delay-tolerant broadcasting. IEEE Trans. Broadcasting 53(1), pp. 369-381 (2007).

27. Karlsson G., Lenders V., May M., Wacha C.: Wireless ad hoc podcasting. In: Proc. IEEE Conf. Sensor, Mesh and Ad Hoc Communications and Networks (SECON), San Diego, CA (2007).

28. Cenceme website. [Online]. Available: http://cenceme.org/

29. Metrosense website. [Online]. Available: http://metrosense.cs.dartmouth.edu/

30. Crawdad website. [Online]. Available: http://crawdad.cs.dartmouth.edu/

31. World Health Organization, World report on road traffic injury prevention, 2004.

32. Vehicle infrastructure integration (vii). [Online]. Available: http://www.its.dot.gov/itsnews/ fact_sheets/vii.htm

33. Dot's intellidrive program. [Online]. Available: http://www.intellidriveusa.org/whoweare/ program.php

34. ETSI intelligent transport systems. [Online]. Available: http://www.etsi.org/WebSite/ Technologies/IntelligentTransportSystems.aspx

35. ITS project web site. [Online]. Available: http://www.its.dot.gov/index.htm

36. ITS standards on the move - etsi. [Online]. Available: http://www.etsi.org/WebSite/document/ Technologies/ETSIpresentations

37. IEEE 802.11 Wireless LAN Working Group, Wlan mac and phy specifications: Wireless access in vehicular environments (wave). Tech. Rep. (2005).

38. IEEE 1609 - family of standards for wireless access in vehicular environments (wave). [Online]. Available: http://www.standards.its.dot.gov/fact_sheet.asp?f=80

39. Gozalvez J., Sepulcre M.: Opportunistic technique for efficient wireless vehicular communications. IEEE Veh. Technol. Mag. 2(4), pp. 33-39 (2007).

40. Gallagher B., Akatsuka H.: Wireless communications for vehicle safety: Radio link performance \& wireless connectivity issues. IEEE Veh. Technol. Mag. 1(4), pp. 4-24 (2006).

41. ITU-internet report 2005: Internet of things. [Online]. Available: http://www.itu.int/osg/spu/ publications/internetofthings/

42. Yan L., Zhang J., Yang L., Ning H. (Eds.): The Internet of Things. From RF-ID to the Next Generation Pervasive Networked Systems. CRC Press, Boca Raton, FL (2008).

43. Atzori L., Iera A., Morabito G.: The internet of things: A survey. Computer Networks 54(15), pp. 2787-2805 (2010).

44. Jacobson V., Smetters D.K., Thornton J.D., Plass M.F., Briggs N.H., Braynard R.L.: Networking named content. In: Proc. ACM Int. Conf. Emerging Networking Experiments and Technologies, Rome, Italy (2009).

45. Taniguchi N.: On the basic concept of nano-technology. In: Proc. Int. Conf. on Production Engineering, London, UK (1974).

46. Suda T., Moore M., Nakano T., Egashira R., Enomoto A.: Exploratory research on molecular communication between nanomachines. In: Proc. Genetic and Evolutionary Computation Conf., Washington, DC (2005).

47. Akyildiz I., Brunetti F., Blázquez C.: Nanonetworks: A new communication paradigm. Computer Networks 52, pp. 2260-2279(2008).

48. Dressler F., Akan O.: A survey on bio-inspired networking. Computer Networks 54, pp. 881900 (2010). 
49. Moore M., Enomoto A., Nakano T., Egashira R., Suda T., Kayasuga A., Kojima H., Sakakibara H., Oiwa K.: A design of a molecular communication system for nanomachines using molecular motors. In: Proc. IEEE Int. Conf. Pervasive Computing and Communications, Pisa, Italy (2006).

50. Nakano T., Suda T., Moore M., EgashiraR., Enomoto A., Arima K.: Molecularcommunication for nanomachines using intercellularcalcium signaling. In: Proc. IEEE Conf. Nanotechnology, Nagoya, Japan (2005).

51. Atakan B., Akan O.: An information theoretical approach for molecular communication. In: Proc. IEEE/ACM Int. Conf. Bio-Inspired Models of Network, Information and Computing Systems, Budapest, Hungary (2007).

52. Atakan B., Akan O.B.: On channel capacity and error compensation in molecular communication. Springer Trans. Computational Systems Biology LNBI 5410, pp. 59-80 (2008).

53. Atakan B., Akan O.: On molecular multiple-access, broadcast, and relay channel in nanonetworks. In: Proc. IEEE/ACM Int. Conf. Bio-Inspired Models of Network, Information and Computing Systems, Hyogo, Japan (2008). 